\title{
Determining insulin sensitivity from glucose tolerance tests in Iberian and landrace pigs
}

\author{
José M Rodríguez-López ${ }^{1}$, Manuel Lachica ${ }^{2}{ }^{2}$ Lucrecia González-Valero ${ }^{2}$, Ignacio Fernández-Fígares ${ }^{\text {Corresp. } 2}$ \\ ${ }^{1}$ Départment Sciences Agronomiques et Animales, Institut Polytechnique LaSalle Beauvais, Beauvais, France \\ 2 Department of Physiology and Biochemistry of Animal Nutrition, Estación Experimental del Zaidín, Consejo Superior de Investigaciones Científicas, \\ Granada, Spain \\ Corresponding Author: Ignacio Fernández-Fígares \\ Email address: ifigares@eez.csic.es
}

As insulin sensitivity may help to explain divergences in growth and body composition between native and modern breeds, metabolic responses to glucose infusion were measured using an intra-arterial glucose tolerance test (IAGTT). Iberian ( $n=4)$ and Landrace $(n=5)$ barrows $(47.0 \pm 1.2 \mathrm{~kg}$ body weight $(B W)$ ), fitted with a permanent carotid artery catheter were injected with glucose (500 mg/kg BW) and blood samples collected at -10, 0, 5, 10, 15, 20, 25, 30, 45, 60, 90, 120 and 180 min following glucose infusion. Plasma samples were analysed for insulin, glucose, lactate, triglycerides, cholesterol, creatinine, albumin and urea. Insulin sensitivity indices were calculated and analysed. Mean plasma glucose, creatinine and cholesterol concentrations were lower $(P<$ 0.01 ) in Iberian (14, 68 and $22 \%$, respectively) than in Landrace pigs during the IAGTT. However, mean plasma insulin, lactate, triglycerides and urea concentrations were greater $(P<0.001)$ in Iberian $(50,35,18$ and $23 \%$, respectively) than in Landrace pigs. Iberian pigs had larger area under the curve $(A \cup C)$ of insulin $(P<0.05)$ or tended to a greater AUC of lactate $(P<0.10)$, and a smaller $(P<0.05)$ AUC for glucose $0-60$ min compared with Landrace pigs. Indices for estimating insulin sensitivity in fasting conditions indicated improved $\beta$-cell function in Iberian compared with Landrace pigs, but no difference $(P>$ $0.10)$ in calculated insulin sensitivity index was found after IAGTT between breeds. A time response $(P<0.05)$ was obtained for insulin, glucose and lactate so that maximum concentration was achieved at 10 and 15 min post-infusion for insulin (Iberian and Landrace pigs, respectively), immediately post-infusion for glucose, and 20 min postinfusion for lactate, decreasing thereafter until basal levels. There was no time effect for the rest of metabolites evaluated. In conclusion, growing Iberian pigs challenged with an IAGTT showed changes in biochemical parameters and insulin response that may indicate an early stage of insulin resistance. 


\section{Determining insulin sensitivity from glucose tolerance tests in Iberian and Landrace pigs}

José Miguel Rodríguez-López ${ }^{1}$, Manuel Lachica², Lucrecia González-Valero², Ignacio Fernández-Fígares ${ }^{2}$

${ }^{1}$ Départment Sciences Agronomiques et Animales, Institut Polytechnique LaSalle Beauvais, Beauvais, France

${ }^{2}$ Department of Physiology and Biochemistry of Animal Nutrition, Estación Experimental del Zaidín, CSIC, Armilla, Granada, Spain

Corresponding Author:

Ignacio Fernández-Fígares ${ }^{2}$

Camino del Jueves s/n, Armilla, Granada, 18100, Spain

Email address: ifigares@eez.csic.es

\section{Abstract}

4 As insulin sensitivity may help to explain divergences in growth and body composition between

5 native and modern breeds, metabolic responses to glucose infusion were measured using an

6 intra-arterial glucose tolerance test (IAGTT). Iberian $(n=4)$ and Landrace $(n=5)$ barrows $(47.0$

$7 \pm 1.2 \mathrm{~kg}$ body weight (BW)), fitted with a permanent carotid artery catheter were injected with

8 glucose (500 mg/kg BW) and blood samples collected at -10, 0, 5, 10, 15, 20, 25, 30, 45, 60, 90, 
9120 and 180 min following glucose infusion. Plasma samples were analysed for insulin, glucose,

10 lactate, triglycerides, cholesterol, creatinine, albumin and urea. Insulin sensitivity indices were

11 calculated and analysed. Mean plasma glucose, creatinine and cholesterol concentrations were

12 lower $(P<0.01)$ in Iberian $(14,68$ and $22 \%$, respectively) than in Landrace pigs during the

13 IAGTT. However, mean plasma insulin, lactate, triglycerides and urea concentrations were

14 greater $(P<0.001)$ in Iberian $(50,35,18$ and 23\%, respectively) than in Landrace pigs. Iberian

15 pigs had larger area under the curve $($ AUC) of insulin $(P<0.05)$ or tended to a greater AUC of

16 lactate $(P<0.10)$, and a smaller $(P<0.05)$ AUC for glucose $0-60$ min compared with Landrace

17 pigs. Indices for estimating insulin sensitivity in fasting conditions indicated improved $\beta$-cell

18 function in Iberian compared with Landrace pigs, but no difference $(P>0.10)$ in calculated

19 insulin sensitivity index was found after IAGTT between breeds. A time response $(P<0.05)$ was

20 obtained for insulin, glucose and lactate so that maximum concentration was achieved at 10 and

2115 min post-infusion for insulin (Iberian and Landrace pigs, respectively), immediately post-

22 infusion for glucose, and 20 min post-infusion for lactate, decreasing thereafter until basal levels.

23 There was no time effect for the rest of metabolites evaluated. In conclusion, growing Iberian

24 pigs challenged with an IAGTT showed changes in biochemical parameters and insulin response

25 that may indicate an early stage of insulin resistance.

\section{Introduction}

28 The Iberian pig is a slow growing native breed of the Mediterranean basin with much greater

29 whole body fat content than lean-type pigs (Nieto et al., 2002). Compared with conventional

30 breeds, Iberian pigs show a lower efficiency of energy utilisation for protein deposition in the

31 growing period (Barea et al., 2007). The greater relative viscera weight (Rivera-Ferre et al., 
32 2005) and total heat production (González-Valero et al., 2016) associated in part with the greater

33 rate of muscle protein turnover (Rivera-Ferre et al., 2005) in Iberian compared with lean-type

34 pigs help to explain the low energy efficiency for growth. In fact, Rivera-Ferre et al. (2005)

35 showed that muscle protein degradation was increased in Iberian pigs resulting in decreased

36 muscle protein accretion compared with Landrace pigs. Interestingly, insulin resistance at the

37 muscle level could explain an increased protein degradation (Wang et al., 2006) affecting overall

38 protein accretion. In a previous study using balanced or lysine deficient diets at two crude protein

39 levels, Iberian had greater fasting serum insulin concentration than Landrace pigs (Fernández-

40 Fígares et al., 2007), suggesting the possibility of insulin resistance in Iberian pigs. We

41 hypothesised that Iberian pigs have decreased insulin sensitivity, which could explain differences

42 on growth, body composition and metabolic characteristics compared with modern breeds. The

43 objective of the present study was to evaluate differences on insulin sensitivity between Iberian

44 and Landrace pigs using an intra-arterial glucose tolerance test (IAGTT).

\section{Materials \& Methods}

\section{Animals and experimental design}

48 All procedures used in this study were approved by the Bioethical Committee of the Spanish

49 Council for Scientific Research (RD 53/2013; CSIC, Spain; project reference RECUPERA 2020 ,

50 FEDER funding) and the animals were cared for in accordance with the Royal Decree No.

51 1201/2005 (Spain). The experiment was performed with five Landrace and four Iberian (Silvela

52 strain) barrows supplied by Granja El Arenal (Córdoba, Spain) and Sánchez Romero Carvajal

53 (Jabugo S.A., Puerto de Santa María, Cádiz, Spain), respectively. 
54 The pigs were group housed in a controlled-environment room $\left(20 \mathrm{~m}^{2} ; 21 \pm 1.5^{\circ} \mathrm{C}\right)$ with $\mathrm{ad}$

55 libitum access to a standard barley-soybean meal diet (160 g crude protein/kg; $14 \mathrm{MJ}$

56 metabolizable energy/kg dry matter) and water. During acclimatization, the pigs were adapted to

57 close contact with the personnel involved in the study to facilitate pig handling without stress.

58 After acclimatization and to subsequently avoid the stress of repeated blood sampling, each

59 animal was surgically fitted with a chronic catheter (Tygon, i.d. $1.02 \mathrm{~mm}$, o.d. $1.78 \mathrm{~mm}$; Cole-

60 Parmer, Vernon Hills, IL, USA) in the carotid artery following a procedure described previously

61 (Rodríguez-López et al., 2013). In brief, Tygon rings were attached to the catheter to mark the

62 extent of introduction into the vessel. The day before surgery, pigs were placed in individual

63 pens $\left(2 \mathrm{~m}^{2}\right)$, where nose and eye contact was possible, in a controlled environment room $(21 \pm$

$64 \quad 1.5^{\circ} \mathrm{C}$ ) and feed and water removed. General anaesthesia was induced using an intamuscular

65 (i.m.) combination of Ketamine (15 mg/kg BW; Imalgene 1000, Merial, Barcelona,

66 Spain)/Azaperone (2 mg/kg BW; Stresnil, Steve, Barcelona, Spain) and maintained with

67 isoflurane (0.5-2\%; Isoflo; Laboratorios Esteve S.A., Barcelona, Spain) and $\mathrm{O}_{2}(22-44 \mathrm{~mL} / \mathrm{kg}$

$68 \mathrm{BW} / \mathrm{min}$ ) through a face mask. N-butyl hyoscine bromide + Sodium metamizol (Buscapina

69 Compositum; Boehringer Ingelheim Spain S.A., Barcelona, Spain) was administered as analgesic

70 (5 mL i.m.). Strict aseptic and sterile conditions were applied along the whole surgical

71 procedure. An incision $(8-10 \mathrm{~cm})$ was done along the jugular furrow and to expose the carotid

72 artery. Catheter was introduced $12 \mathrm{~cm}$ toward the aorta arch and fixed by non-absorbable suture.

73 The catheter was secured directly in place with a purse-string suture where the artery was not

74 occluded. The incision was sutured. A patch $(10 \times 10 \mathrm{~cm})$ was glued to the skin together with the

75 catheter close to the exteriorization point (down and caudal from the ear), guided to the shoulder,

76 fixed again and kept coiled with a second patch-pocket. Following surgery, pigs returned to their 
77 individual pens under heat lamps to provide additional warmth during anaesthesia recovery.

78 After that, pigs were fed with free access to water. Feed, water intake and body temperature were

79 monitored during a couple of days. Then, pigs were group housed again until the blood sampling 80 and fed at $2.4 \times$ metabolizable energy for maintenance $\left(444 \mathrm{~kJ} / \mathrm{kg}^{0.75}\right.$ body weight $(\mathrm{BW}) /$ day;

81 National Research Council (NRC), 1998). Wound from surgery and catheter exteriorization site

82 were kept clean and sprayed with antibiotic (Veterin Tenicol; Lab. Intervet S.A., Salamanca,

83 Spain) to prevent infection. Pigs were injected i.m. with a broad spectrum antibiotic (Duphapen

84 Strep; Fort Dodge Vet. S.A., Gerona, Spain) during 5 days (5-10 mg/kg BW/day). After 10 days,

85 stitches were removed and pigs were ready for the blood sampling. Patency of catheter was

86 checked weekly, cleaned with alcohol and flushed with sterile heparinized (Fragmin, 5000

87 IU/0.2 mL; Pharmacia Spain S.A., Barcelona, Spain) saline (250 IU/mL).

88 The day before the experiment, pigs were randomly accommodated in the individual pens

89 for easier blood sampling and fed normally. On the day of the experiment, all pigs ( $46.0 \pm 3.0$

90 and $47.8 \pm 3.6 \mathrm{~kg} \mathrm{BW}$ for Iberian and Landrace pigs, respectively; that is about 18 and 14 weeks

91 of age, respectively) were given an intra-arterial bolus ( $500 \mathrm{mg} / \mathrm{kg} \mathrm{BW})$ of glucose ( $50 \%$ sterile

92 dextrose; glucosado 50\% Braun, B. Braun Medical S.A., Rubi, Barcelona, Spain) over one min

93 period after an overnight fast. The catheter was immediately flushed with $5 \mathrm{~mL}$ of sterile saline

94 solution. Blood samples $(5 \mathrm{~mL})$ were collected at $-10,0$ (20-30 seconds after the bolus of glucose

95 and the saline solution), 5, 10, 15, 20, 25, 30, 45, 60, 90, 120 and 180 min following glucose

96 infusion. At the end of the study, pigs were slaughtered -in accordance with the Royal Decree

97 No. 1201/2005 (Spain)- by electrical stunning.

98 The staff involved in the experiment was aware of the group allocation at the different stages 99 of the experiment. 


\section{Biochemical analysis and calculations}

102 Plasma was obtained by centrifugation $\left(4^{\circ} \mathrm{C}, 1820 \times \mathrm{g}\right.$ for $30 \mathrm{~min}$; Eppendorf $5810 \mathrm{R}$, Hamburg,

103 Germany) and stored in aliquots at $-20^{\circ} \mathrm{C}$ until insulin and metabolites (glucose, lactate,

104 triglycerides, cholesterol, creatinine, albumin and urea) were analysed. All samples were assayed

105 in duplicate except for insulin which was assayed in triplicate.

106 Insulin was measured using commercially-available radioimmuno assay kit following the

107 directions of the manufacturer (Millipore porcine insulin radioimmuno assay kit; Cat. PI-12K).

108 Radioactivity in samples was measured using a gamma counter (Behring 1612; Nuclear

109 Enterprises Ltd, Edinburgh, Scotland). Human insulin was used as standard, and the assay was

110 validated for use in porcine plasma samples (Fernández-Fígares et al., 2007). The intra- and

111 inter-assay coefficient of variation for plasma insulin were 4.4 and $9.1 \%$, respectively. The

112 lowest level of insulin that can be detected by this assay is $1.611 \mu \mathrm{U} / \mathrm{mL}$ when using a $100 \mu \mathrm{L}$

113 sample size.

114 Plasma glucose, lactate, triglycerides, cholesterol, creatinine, albumin and urea were

115 measured colorimetrically using an automated Cobas Integra 400® analyser (Roche Diagnostics

$116 \mathrm{GmbH}$, Mannheim, Germany). Analyses were performed in a single run where intra-assays

117 coefficients of variation were $1.3,0.92,1.6,0.81,3.1,1.2$ and $2.3 \%$ for glucose, lactate,

118 triglycerides, cholesterol, creatinine, albumin and urea, respectively.

119 Responses of plasma insulin, glucose and lactate were evaluated separately by computing

120 total area under the response curve (AUC) determined using trapezoidal geometry (GraphPad

121 Prism, Version 5.02. San Diego, CA) for the time period indicated following intra-arterial

122 glucose infusion (e.g. AUC0-5 stands for the integrated area between 0-5 min post-infusion, 
123 AUC0-10 between 0-10 min post-infusion, and so on, until AUC0-180). Basal levels per breed

124 (at time $-10 \mathrm{~min}$ ) were used to calculate the corresponding AUC per metabolite. The rates of

125 decline in plasma insulin and glucose concentrations for both breeds were calculated based on

126 the slope in the linear portion of the response curve from 0 to $30 \mathrm{~min}$ after IAGTT challenge

127 (Christoffersen et al., 2009). Results were then expressed as a fractional rate constant determined

128 from the slope of the natural logarithm of plasma concentrations vs. time (Shipley and Clark,

1291972 (cited by Gopinath and Etherton, 1989)). The fractional turnover rates $(k)$, or disappearance

130 rates, of plasma insulin and glucose $(\% / \mathrm{min})$ were calculated using the relationship (Kaneko et

131 al., 2008):

$132 k=(\operatorname{Ln} 1-\operatorname{Ln} 2) /\left(\mathrm{T}_{2}-\mathrm{T}_{1}\right)$

133 where $\operatorname{Ln} 1$ and $\operatorname{Ln} 2$ are the natural logarithms of plasma insulin $(\mu \mathrm{U} / \mathrm{mL})$ or glucose $(\mathrm{Mm})$

134 concentrations at times $\mathrm{T}_{1}(0 \mathrm{~min})$ and $\mathrm{T}_{2}(30 \mathrm{~min})$, respectively.

135 From the $k$ value, the half-life, $\mathrm{T}_{1 / 2}(\mathrm{~min})$, may be calculated as:

$136 \mathrm{~T}_{1 / 2}=100 \times 0.693 / k$

137 For insulin sensitivity, indices used in human medicine were used.

138 The so-called homeostasis model assessment (HOMA; Matthews et al., 1985) was

139 calculated for estimating insulin resistance (HOMA-IR) and $\beta$-cell function (HOMA-\%B) at

140 fasting conditions, as follows:

141 HOMA-IR $=$ fasting plasma insulin $(\mu \mathrm{U} / \mathrm{mL}) \times$ fasting plasma glucose $(\mathrm{mM}) / 22.5$

142 HOMA- $\% \mathrm{~B}=(20 \times$ fasting plasma insulin $(\mu \mathrm{U} / \mathrm{mL})) /($ fasting plasma glucose $(\mathrm{mM})-3.5)$

143 It is assumed that non-insulin-resistant individuals have $100 \% \beta$-cell function and an insulin 144 resistance of 1. 
146 as:

147 QUICKI $=1 /\left[\operatorname{Ln}\left(\mathrm{I}_{0}\right)+\operatorname{Ln}\left(\mathrm{G}_{0}\right)\right]$

148 where $\mathrm{I}_{0}$ is the fasting insulin $(\mu \mathrm{U} / \mathrm{mL})$, and $\mathrm{G}_{0}$ is the fasting glucose $(\mathrm{mg} / \mathrm{dl})$.

149 Finally, the insulin sensitivity index (CSI; Tura et al., 2010) was calculated as:

$150 \quad \mathrm{CSI}=K_{G} /\left(\Delta \mathrm{AUC}_{\mathrm{INS}} / \mathrm{T}\right)$

151 where $K_{G}$ is the slope of Ln glucose in the linear portion of the response curve, $\Delta \mathrm{AUC} \mathrm{INS}_{\text {is }}$ the

152 AUC of insulin above basal value, and $\mathrm{T}$ is the time interval (between 0 and $30 \mathrm{~min}$ ) when $K_{G}$

153 and $\triangle \mathrm{AUC}_{\mathrm{INS}}$ are calculated.

155 Statistical analyses

156 The number of animals was a priori calculated using the $\mathrm{G}^{*}$ Power software (Heinrich-Heine-

157 Universität Düsseldorf (Faul et al., 2007)). Accepting an alpha risk of 0.05 and a beta risk of 0.2

158 in a two-sided test, five subjects are necessary in first group and five in the second to recognize

159 as statistically significant a difference greater than or equal to $12 \mu \mathrm{U} / \mathrm{mL}$ on insulin

160 concentration and a common standard deviation of $6.3 \mu \mathrm{U} / \mathrm{mL}$ based on previous studies

161 (Fernández-Fígares et al., 2007). A total of five pigs per treatment was also used by others (e.g.

162 Stoll et al., 1999). However, one Iberian pig lost the arterial catheter during the recovery period

163 after surgery and only four Iberian pigs could be used.

164 Plasma metabolites were evaluated using a mixed ANOVA with repeated measures (Version

165 9.4; PROC MIXED, SAS Institute Inc., Cary, NC, USA) with the fixed effects of breed, time of

166 sampling and their interaction in the model statement. The pig was considered the experimental

167 unit and a random effect. First-order ante dependence covariance ANTE(1) was used, which 
168 allows unequal variances over time and unequal correlations and covariance among different

169 pairs of measurements. Plasma concentration differences between breeds at each sampling time

170 were analysed by the pdiff (piecewise differentiable) option.

171 Assumptions that are required for an ANOVA were tested following the protocol from Zuur

172 et al. (2010). Homogeneity of variance was assured by applying the Levene's-Test. No

173 transformation was required. Least square means and pooled standard error of mean (SEM) are

174 presented. Outliers were identified and removed when the absolute studentized residues

175 exceeded 3. Differences were considered significant at $P<0.05$ and trends approaching

176 significance were considered for $0.05<P<0.10$.

\section{Results}

179 Average plasma metabolites and insulin concentrations after the IAGTT are shown in Table 1.

180 Mean plasma glucose, cholesterol and creatinine concentrations were lower in Iberian (14, 22

181 and $68 \%$, respectively; $P<0.05)$ compared with Landrace pigs. However, mean plasma insulin,

182 lactate, triglycerides and urea concentrations were greater in Iberian $(50,35,18$ and 23\%,

183 respectively; $0.01<P<0.001)$ than in Landrace pigs. No differences $(P>0.10)$ were found

184 between breeds for albumin levels.

185 Fasting plasma insulin was greater in Iberian compared with Landrace pigs $(P<0.05$; Table

186 2) whereas fasting plasma glucose was similar for both breeds $(P>0.10$; Table 2$)$. No

187 differences between breeds were found in fasting plasma albumin (Iberian 0.50 and Landrace

$1880.54 \mu \mathrm{M}$ ), urea (Iberian 3.3 and Landrace $3.0 \mathrm{mM}$ ), cholesterol (Iberian 1.46 and Landrace 1.79

$189 \mathrm{mM}$ ) and triglycerides (Iberian 0.28 and Landrace $0.22 \mathrm{mM}$ ). On the other hand plasma fasting 
190 creatinine was lower in Iberian pigs compared to Landrace (54 and $102 \mu \mathrm{M}, \mathrm{SEM}=8.18$,

191 respectively; $P<0.01)$.

192 Only plasma insulin (Fig. 1), glucose (Fig. 2) and lactate (Fig. 3) concentrations changed

193 throughout time $(P<0.001$; Table 1) after the IAGTT.

194 An interaction between breed and time was found for plasma insulin, such that concentration

195 of insulin was greater in Iberian pigs from -10-15 min and from 90-180 $\min (P<0.05$, with $P<$

1960.10 at times 0 and $90 \mathrm{~min})$ and lower at $25 \mathrm{~min}(P<0.10$; Fig. 1$)$. In both breeds, plasma

197 insulin levels increased 7-fold, reaching a peak concentration at 10 and 15 min after glucose

198 infusion for Iberian $(113.6 \pm 7.1 \mu \mathrm{U} / \mathrm{mL})$ and Landrace $(55.7 \pm 6.4 \mu \mathrm{U} / \mathrm{mL})$ pigs, respectively.

199 Insulin remained well above fasting levels until 20 and $45 \mathrm{~min}$ after glucose infusion for Iberian

200 and Landrace pigs, respectively; thereafter insulin levels rapidly decreased until fasting levels

201 were attained. Insulin disappearance rate tended to increase in Iberian compared with Landrace

202 pigs $(0.05<P<0.10)$ while insulin half-life tended to decrease $(0.05<P<0.10$; Table 2$)$.

203 Glucose peaked (Fig. 2) immediately after glucose infusion reaching a value of 19.6 and

$20421.2 \mathrm{mmol} / \mathrm{L}$ for Iberian and Landrace pigs, respectively. Subsequently, glucose concentration

205 gradually decreased to values below fasting levels after 25 and 30 min, respectively for Iberian

206 and Landrace pigs. The lowest plasma glucose concentration (glucose nadir) was found at 45

$207 \mathrm{~min}$ (2.95 and $3.70 \mathrm{mmol} / \mathrm{L}$ for Iberian and Landrace pigs, respectively). After glucose nadir,

208 glucose concentration gradually increased again to reach values comparable to fasting levels at

$209180 \mathrm{~min}$. No differences were found between breeds for glucose disappearance rate $(P>0.10$;

210 Table 2) or glucose half-life $(P>0.10$; Table 2).

211 Lactate increased after the IAGTT, peaked at $20 \mathrm{~min}$ for both breeds and declined

212 progressively until reaching basal concentrations at $180 \mathrm{~min}$ (Fig. 3). 
213 The AUC values for each sampling time of insulin, glucose and lactate are shown in Table 3,

2144 and 5, respectively. Insulin AUC was greater $(P<0.05)$ for Iberian compared with Landrace

215 pigs at all times.

216 Conversely, glucose AUC at 0-15, 0-20, 0-25, 0-30, 0-45 and 0-60 min were lower $(P<$

217 0.05) for Iberian than Landrace pigs. Aditionally, glucose AUC tended to be lower $(0.05<P<$

$2180.10)$ at $0-10,0-90$ and $0-120 \mathrm{~min}$. Plasma lactate AUC was greater $(P<0.05)$ for Iberian pigs at

$2190-10$ and $0-15 \mathrm{~min}$ and tended to be greater $(0.05<P<0.10)$ at $0-5,0-20,0-25,0-90,0-120,0-$

$220 \quad 150$ and 0-180 min after glucose infusion.

221 Indices of insulin sensitivity are shown in Table 2. The QUICKI index decreased $(P<0.05)$

222 while HOMA-\%B index increased $(P<0.01)$ in Iberian compared with Landrace pigs. No

223 differences $(P>0.10)$ were found for HOMA-IR and CSI.

224

225 Discussion

226 The IAGTT method allowed the comparison of the insulin responsiveness of an obese (Iberian)

227 and a lean (Landrace) pig breed. It is well established that Iberian pigs have a much greater

228 capacity of lipid deposition in comparison to lean swine breeds (Nieto et al., 2002; Ovilo et al.,

229 2005; Muñoz et al., 2009) and has since been proposed as a pig model for obesity studies

230 (Rodriguez Rodriguez et al., 2020). For the reason that important differences in for instance

231 protein turnover may take place during development (Lobley, 1993), the study of animal breeds

232 with disparate growth capability is not a simple issue. Thus, it is desirable that animals are

233 comparable for age or physiological state. As the developmental age of the animals may vary, a

234 decision was made regarding the use pigs of the same BW considering that age difference at this

235 early state was acceptable.

Peer] reviewing PDF | (2020:10:54158:2:0:NEW 22 Jan 2021) 
As arterial blood represents the metabolites concentration to which the tissues are exposed

237 (Brouns et al., 2005), chronic catheters were inserted in carotid artery for glucose infusion and

238 blood sampling.

239 In the current study we have shown that despite the higher fasting plasma insulin, Iberian

240 pigs produce a higher insulin response after glucose infusion when compared to Landrace pigs

241 (18 and 14 weeks of age, respectively). Greater postprandial serum levels of insulin have been

242 described in $20 \mathrm{~kg}$ BW Iberian (11 weeks of age) compared to Landrace pigs after glucose

243 infusion (Fernández-Fígares et al., 2007), and in $11 \mathrm{~kg} \mathrm{BW}$ Ossabaw (obese; 10 weeks of age)

244 compared to $16.5 \mathrm{~kg}$ Yorkshire (10 weeks of age) pigs (Wangsness et al., 1981). However, other

245 comparative studies using a standard diet found increased insulin secretion in $75-120 \mathrm{~kg} \mathrm{BW}$

246 Large White boars than in 40-75 kg BW Meishan boars (obese breed) at 20 and 52 weeks of age,

247 respectively (Weiler et al., 1998). The limited growth and development of slow growing pigs

248 could result at least partly from disturbances in insulin secretion and/or in insulin binding,

249 leading to insulin sensitivity, because most cells of the body require insulin for adequate uptake

250 of glucose and amino acids (Claus and Weiler, 1994). If the concentration of insulin is compared

251 among animals of different breeds, the sensitivity of each breed to insulin should be considered.

252 In this study we were also interested in other key metabolites which could provide additional

253 information concerning reduced insulin sensitivity in Iberian pigs. After glucose infusion,

254 glucose plasma concentration rapidly returned to preprandial values in the present experiment,

255 which indicates that exogenous glucose was efficiently metabolized, stored as glycogen, or both.

256 As expected, when glucose was infused, plasma glucose levels were rapidly increased and a

257 subsequent insulin response was observed. The elevated insulin lowered plasma glucose below

258 fasting values within 20 and 25 min for Iberian and Landrace pigs, respectively, and insulin 
259 levels returned to baseline as plasma glucose declined. In our study, glucose concentration and

260 glucose AUC during the IAGTT were lower in Iberian compared with Landrace pigs, with no

261 differences in fasting plasma glucose, maybe due to the limited number of pigs. When

262 interpreting the individual glucose curves, a monophasic pattern was identified for both breeds.

263 The lower glucose AUC of Iberian pigs ( $-19 \%$ on average) may be related to the greater insulin

264 AUC ( $+33 \%$ on average), a common pattern in many models of obesity (Kay et al., 2001).

265 However, the reasons for the unequal physiological response between breeds are not well

266 understood and must be discussed.

267 As it has been proved that the energy needs of portal-drained viscera are fulfilled by the

268 oxidation of glucose, glutamate, and glutamine in pigs (Stoll et al., 1999), a larger

269 gastrointestinal tract of Iberian pigs compared to Landrace (Rivera-Ferre et al., 2005) is in line

270 with the decreased AUC of glucose reported in our experiment.

271 However, despite the larger size of the gastrointestinal tract and lower portal blood flow

272 (González-Valero et al., 2016) of Iberian compared with Landrace pigs, no differences on net

273 portal flux of glucose after ingestion of the same diet were found (Rodríguez-López et al., 2013).

274 Differences on insulin stimulated glucose transport at portal-drained viscera level may help to

275 explain these results. Iberian have lower glucose concentrations than Landrace pigs after an

276 intravenous adrenaline challenge (Fernández-Fígares et al., 2016), suggesting a decreased

277 response of Iberian pigs to sympathetic nervous system stimuli which is in line with the lower 278 glucose AUC reported here.

279 When insulin sensitivity indices used in human medicine were applied to the conditions of 280 the present experiment, QUICKI and HOMA-\%B were more sensitive detecting differences

281 between breeds. Indeed, QUICKI index decreased in Iberian compared with Landrace pigs, 
282 pointing out an incipient insulin sensitivity impairment in fasting Iberian pigs. Similarly, reduced

283 QUICKI index (0.5 vs. 0.6) was found in Bama miniature pigs fed a high sucrose and fat diet

284 compared with a control diet, respectively (Liu et al., 2017). The QUICKI index has been shown

285 to provide reasonable approximations of insulin efficiency in minipigs (Christoffersen et al., 286 2009).

287 When we used the HOMA, differences on hepatic HOMA-IR were negligible between 288 breeds (3.3 and 2.3 for Iberian and Landrace pigs, respectively; $P>0.10$ ). However, Iberian had 289 improved $\beta$-cell function compared with Landrace pigs according to HOMA-\%B (267 and 100, 290 respectively; $P<0.01$ ), which may be due to enhanced sensitivity of the $\beta$-cells to glucose 291 during the fasting period. As a consequence, $\beta$-cell insulin synthesis in Iberian pigs increased in 292 accordance with the increased insulin release after the glucose tolerance test and the elevated 293 basal insulin concentrations reported for Iberian pigs. This is consistent with decreased QUICKI 294 in Iberian pigs compared to Landrace ( 0.31 and 0.33 , respectively; $P<0.05)$.

295 Lactate appearance after an intravenous glucose test is positively associated with insulin 296 sensitivity in humans (Lovejoy et al., 1992), as it is related to lactate production by insulin 297 sensitive tissues (mainly muscle and fat). Because only limited amounts of lactate are produced 298 by muscle after glucose loading (Ykijarvinen et al., 1990), the source of lactate appearance 299 should predominantly be adipose tissue (Lovejoy et al., 1992), with a large capacity to convert 300 glucose to lactate (Marin et al., 1987). We report here a delay of $20 \mathrm{~min}$ in plasma lactate 301 elevation relative to glucose peak following IAGTT, which may reflect the time lag in adipose 302 tissue uptake of glucose and subsequent lactate production under the stimulation of insulin.

303 Compared with Landrace, the increased lactate AUC in Iberian pigs after the IAGTT could 304 therefore be a consequence of the greater adipose tissue availability (Nieto et al., 2002) instead 
305 of greater insulin sensitivity. On the other hand, insulin resistance was associated with elevated

306 basal lactate levels in obese humans (Lovejoy et al., 1990), so increased basal lactate

307 concentrations in Iberian pigs ( 1.040 vs. $0.730 \mathrm{mmol} / \mathrm{L} ; \mathrm{SEM}=0.063)$ could also indicate

308 insulin resistance or reduced insulin sensitivity. Although inhibition of insulin action on

309 glycogenolysis in fasting conditions may lead to increased glucose release from glycogen and

310 subsequent conversion of glucose to lactate, there is no direct evidence of this. There is indirect

311 evidence, though, that elevated lactate levels could be a consequence of greater adipose tissue

312 availability and may also reflect a glucose sparing effect (decreased glucose utilisation) in

313 muscle (Pearce and Connett, 1980).

314 Obesity is frequently associated with different degrees of dyslipidemia manifested as

315 increased triglyceridemia and low HDL-cholesterol. In our experiment, we found lower plasma

316 total cholesterol but greater plasma triglycerides concentration in Iberian compared with

317 Landrace pigs. Although we did not separate LDL and HDL fractions, total cholesterol

318 concentration are phenotypically related with LDL and HDL cholesterol concentrations in pigs

319 (Rauw et al., 2007). Reduced total cholesterol concentration could be due to reduced hepatic

320 insulin sensitivity as insulin stimulates cholesterol synthesis (Nelson and Cox, 2017). In any case

321 the cholesterolemia for both breeds in the present experiment was in the lower range of

322 published values (Fernández-Fígares et al., 2007). Indeed, the pigs in this study were still very

323 young and so a greater level should be expected at a later stage of development (Rauw et al.,

324 2007).

325 Previous studies in our lab have shown the low genetic potential of growing Iberian pigs for 326 muscle protein deposition in comparison to lean breeds (Nieto et al., 2002), possibly due to the

327 greater muscle protein degradation and turnover of the former (Rivera-Ferre et al., 2005). In line 
328 with this, plasma urea level (an indirect protein degradation indicator) was in the present study

$32923 \%$ greater in Iberian compared with Landrace pigs. Differences on circulating insulin or the

330 capacity of insulin release between breeds may explain differences in lean tissue deposition, as

331 insulin has an important role in skeletal muscle metabolism (Wang et al., 2006). In obese $\mathrm{db} / \mathrm{db}$

332 mice (a model of insulin deficiency) higher muscle protein degradation in comparison with

333 control mice (normal plasma insulin concentration) was reported; the authors concluded that

334 insulin resistance was associated with accelerated muscle protein degradation (Wang et al.,

335 2006). The elevated protein degradation reported in Iberian compared with Landrace pigs

336 (Rivera-Ferre et al., 2005) suggests the possibility of insulin resistance at this level. The lower

337 plasma creatinine level (indicator of muscle mass) found in this study for Iberian pigs is in

338 accordance with previous studies (Fernández-Fígares et al., 2007) and also with the low muscle

339 protein deposition and muscle size described previously (Nieto et al., 2002; Rivera-Ferre et al.,

340 2005). As insulin resistance is associated with decreased muscle mass, plasma creatinine levels

341 can also be used as an indicator of insulin signalling disorders as reported by Kashima et al.

342 (2017) in humans. Further research regarding amino acids concentration after an IAGTT may

343 help to explain differences in the effect of insulin on muscle protein metabolism between breeds.

344 Previous studies from our lab indicate that growing Iberian pigs are prone to insulin

345 resistance compared with modern breeds as denoted by increased hepatic gluconeogenesis

346 (González-Valero et al., 2014), greater plasma free fatty acid concentration (Fernández-Fígares

347 et al., 2016) and lower plasma creatinine and QUICKI (Fernández-Fígares et al., 2007).

348 Additionally, in this experiment we show greater HOMA-\%B and increased plasma insulin and

349 lactate concentrations after an IAGTT. The increased plasma insulin AUC after an IAGTT

350 suggests insulin resistance in comparison to the values obtained for lean pigs, although the 
351 concentration of glucose remained low which could indicate the absence of a peripheral insulin

352 resistance. Although Iberian pigs may be considered an obese breed in terms of body

353 composition (Nieto et al., 2002; Barea et al., 2007), insulin resistance mechanisms have not yet

354 been fully established at the development stage of the pigs in this experiment. Insulin resistance

355 and impaired glucose tolerance has been shown in Iberian sows (2.5 years old) ad libitum fed a

356 saturated fat enriched diet for three months (Torres-Rovira et al., 2012).

\section{Conclusions}

359 Although our results support the existence of an insulin resistance or a decreased insulin 360 sensitivity in growing Iberian pigs, caution should be taken because of the reduced number of

361 pigs used. The utilization of the hyperinsulinemic euglycemic clamp, the most definitive

362 approach to determine whole-body insulin action should provide conclusive evidence regarding

363 the establishment of insulin resistance in growing Iberian pigs.

364

\section{Acknowledgements}

366 The authors thank the company Sánchez Romero Carvajal (Jabugo S.A., Puerto de Santa María,

367 Spain) for their helpful collaboration, Dr Luis Lara for statistical advice and Dr Thomas J.

368 Caperna for critically reading the manuscript. A preprint version of this manuscript has been

369 peer-reviewed and recommended by Peer Community In Animal Science

370 (https://doi.org/10.24072/pci.animsci.100004).

371

372

\section{References}


373 Barea R, Nieto R, Aguilera JF. 2007. Effects of the dietary protein content and the feeding level 374 on protein and energy metabolism in Iberian pigs growing from 50 to $100 \mathrm{~kg}$ body weight. Animal 1,357-365 DOI 10.1017/S1751731107666099.

376

377

378

379

380

381

382

383

384

385

386

387

388

389

390

391

392

393

394

395

Brouns F, Bjorck I, Frayn KN, Gibbs AL, Lang V, Slama G, Wolever TMS. 2005. Glycaemic index methodology. Nutrition research reviews 18, 145-171 DOI 10.1079/NRR2005100.

Christoffersen B, Ribel U, Raun K, Golozoubova V, Pacini G. 2009. Evaluation of different methods for assessment of insulin sensitivity in Gottingen minipigs: introduction of a new, simpler method. American Journal of Physiology-Regulatory Integrative and Comparative Physiology 297, R1195-R1201 DOI 10.1152/ajpregu.90851.2008.

Claus R, Weiler U. 1994. Endocrine regulation of growth and metabolism in the pig - a review. Livestock Production Science 37, 245-260 DOI 10.1016/0301-6226(94)90120-1.

Faul, F, Erdfelder E, Lang AG, Buchner A. 2007. G*Power 3: A flexible statistical power analysis program for the social, behavioral, and biomedical sciences. Behavior Research Methods 39, 175-191 DOI 10.3758/bf03193146.

Fernández-Fígares I, Lachica M, Nieto R, Rivera-Ferre MG, Aguilera JF. 2007. Serum profile of metabolites and hormones in obese (Iberian) and lean (Landrace) growing gilts fed balanced or lysine deficient diets. Livestock Science 110, 73-81 DOI 10.1016/j.livsci.2006.10.002.

Fernández-Fígares I, Rodríguez-López JM, González-Valero L, Lachica M. 2016. Metabolic responses to adrenaline challenge in Iberian compared to Landrace pigs. In 9th International Symposium on Mediterranean Pig, Portalegre, Portugal, p. 67.

González-Valero L, Rodríguez-López JM, Lachica M, Fernández-Fígares I. 2014. Metabolic differences in hepatocytes of obese and lean pigs. Animal 8, 1873-1880 DOI 10.1017/S1751731114001748. 
396 González-Valero L, Rodríguez-López JM, Lachica M, Fernández-Fígares I. 2016. Contribution

397 of portal-drained viscera to heat production in Iberian gilts fed a low protein diet:

398 comparison to Landrace. Journal of the Science of Food and Agriculture 96, 1202-1208 DOI

$399 \quad 10.1002 /$ jsfa.7206.

400 Gopinath, R., Etherton TD. 1989. Effects of porcine growth-hormone on glucose-metabolism of 401 pigs .2. Glucose-tolerance, peripheral tissue insulin sensitivity and glucose kinetics. Journal 402 of Animal Science 67, 689-697 DOI 10.2527/jas1989.673689x.

403 Kaneko JJ. 2008. Carbohydrate metabolism and its diseases. In: Clinical biochemistry of 404 domestic animals. $6^{\text {th }}$ edition; Kaneko JJ, Harvey JW and Bruss ML Eds.; Academic Press, $405 \quad$ London, UK; pp. 45-80.

406 Kashima S, Inoue K, Matsumoto M, Akimoto K. 2017. Low serum creatinine is a type 2 diabetes 407 risk factor in men and women: The Yuport Health Checkup Center cohort study. Diabetes and Metabolism 43, 460-464 DOI 10.1016/j.diabet.2017.04.005.

409 Katz A, Nambi S, Mather K, Baron A, Follmann D, Sullivan G, Quon M. 2000. Quantitative

410 insulin sensitivity check index: A simple, accurate method for sssessing insulin sensitivity in

411 humans. Journal of Clinical Endocrinology and Metabolism 85, 2402-2410 DOI

$412 \quad 10.1210 /$ jcem.85.7.6661.

413 Kay JP, Alemzadeh R, Langley G, D'Angelo L, Smith P, Holshouser S. 2001. Beneficial effects

414 of metformin in normoglycemic morbidly obese adolescents. Metabolism-Clinical and

$415 \quad$ Experimental 50, 1457-1461 DOI 10.1053/meta.2001.28078.

416 Liu YQ, Yuan JF, Xiang L, Zhao YQ, Niu MM, Dai X, Chen H. 2017. A high sucrose and high

417 fat diet induced the development of insulin resistance in the skeletal muscle of Bama 

miniature pigs through the Akt/GLUT4 pathway. Experimental Animals 66, 387-395 DOI 10.1538/expanim.17-0010.

Lobley GE. 1993. Species comparisons of tissue protein metabolism: Effects of age and hormonal action. Journal of Nutrition 123, 337-343 DOI 10.1093/jn/123.suppl_2.337.

Lovejoy J, Mellen B, Digirolamo M. 1990. Lactate generation following glucose-ingestion relation to obesity, carbohydrate-tolerance and insulin sensitivity. International Journal of Obesity $14,843-855$.

Lovejoy J, Newby FD, Gebhart SSP, Digirolamo M. 1992. Insulin resistance in obesity is associated with elevated basal lactate levels and diminished lactate appearance following intravenous glucose and insulin. Metabolism-Clinical and Experimental 41, 22-27 DOI 10.1016/0026-0495(92)90185-d.

Marin P, Rebuffescrive M, Smith U, Bjorntorp P. 1987. The glucose-uptake in human adiposetissue. Metabolism-Clinical and Experimental 36, 1154-1160 DOI 10.1111/j.13652362.1971.tb00559.x.

Matthews DR, Hosker JP, Rudenski AS, Naylor BA, Treacher DF, Turner RC. 1985. Homeostasis model assessment - insulin resistance and beta-cell function from fasting plasma-glucose and insulin concentrations in man. Diabetologia 28, 412-419 DOI population analyses of two experimental pig crosses to confirm quantitative trait loci on SSC6 and LEPR effects on fatness and growth traits. Journal of Animal Science 87, 459468. DOI 10.2527/jas.2008-1127. 
440 National Research Council (NRC). 1998. Nutrient requirements of swine, 10th edition. National $441 \quad$ Academy Press, Washington, DC.

442 Nelson DL, Cox MM. 2017. Cholesterol, Steroids, and Isoprenoids: Biosynthesis, Regulation, 443 and Transport. In Lehninger principles of biochemistry. 7th ed.; W.H. Freeman; New York, $444 \quad$ NY; pp.816-832.

445 Nieto R, Lara L, Barea R, Garcia-Valverde R, Aguinaga MA, Conde-Aguilera JA, Aguilera JF. 446 2012. Response analysis of the Iberian pig growing from birth to $150 \mathrm{~kg}$ body weight to 447 changes in protein and energy supply. Journal of Animal Science 90, 3809-3820 DOI $448 \quad$ 10.2527/jas.2011-5027.

449 Nieto R, Miranda A, Garcia MA, Aguilera JF. 2002. The effect of dietary protein content and 450 feeding level on the rate of protein deposition and energy utilization in growing Iberian pigs 451 from 15 to $50 \mathrm{~kg}$ body weight. British Journal of Nutrition 88, 39-49 DOI 10.1079/BJN2002591.

453 Ovilo C, Fernandez A, Noguera JL, Barragan C, Leton R, Rodriguez C, Mercade A, Alves E, 454 Folch JM, Varona L, Toro M. 2005. Fine mapping of porcine chromosome 6 QTL and 455 LEPR effects on body compositionin multiple generations of an Iberian by Landrace 456 intercross. Genetics Research 85, 57-67 DOI 10.1017/S0016672305007330.

457 Pearce FJ, Connett RJ. 1980. Effect of lactate and palmitate on substrate utilization of isolated rat 458 soleus. American Journal of Physiology 238, C149-C159 DOI 10.1152/ajpcell.1980.238.5.C149.

460 Rauw WM, Portoles O, Corella D, Soler J, Reixach J, Tibau J, Prat JM, Diaz I, Gomez-Raya L. 461 2007. Behaviour influences plasma levels in a pig model. Animal 1, 865-871 DOI $462 \quad 10.1017 / \mathrm{S} 175173110700018$. 
463 Rivera-Ferre MG, Aguilera JF, Nieto R. 2005. Muscle fractional protein synthesis is higher in

464 Iberian than in landrace growing pigs fed adequate or lysine-deficient diets. Journal of

465 Nutrition 135, 469-478 DOI 10.1093/jn/135.3.469.

466 Rodríguez-López JM, Lachica M, González-Valero L, Fernández-Fígares I. 2013. Approaches

467 for quantifying gastrointestinal nutrient absorption and metabolism in a native and a modern

468 pig breed. Journal of Agricultural Science 151, 434-443 DOI 10.1017/S0021859612000615.

469 Rodriguez Rodriguez R, Gonzalez-Bulnes A, Garcia-Contreras C, Rodriguez-Rodriguez AE,

470 Astiz S, Vazquez-Gomez M, Pesantez JL, Isabel B, Salido-Ruiz E, González J, Donate

471 Correa J, Luis-Lima S, Porrini E. 2020. The Iberian pig fed with high-fat diet: a model of

472 renal disease in obesity and metabolic syndrome. International Journal of Obesity 44, 457-

$473 \quad 465$ DOI $10.1038 / \mathrm{s} 41366-019-0434-9$.

474 Shipley RA, Clark RE. 1972. Tracer methods for In vivo kinetics. Theory and applications.

$475 \quad$ Academic Press, New York.

476 Stoll B, Burrin DG, Henry J, Yu H, Jahoor F, Reeds PJ. 1999. Substrate oxidation by the portal

477 drained viscera of fed piglets. American Journal of Physiology-Endocrinology and

$478 \quad$ Metabolism 277, E168-E175 DOI 10.1152/ajpendo.1999.277.1.E168.

479 Torres-Rovira L, Astiz S, Caro A, Lopez-Bote C, Ovilo C, Pallares P, Perez-Solana ML,

480 Sanchez-Sanchez R, Gonzalez-Bulnes A. 2012. Diet-induced swine model with

481 obesity/leptin resistance for the study of metabolic syndrome and type 2 diabetes. Scientific

$482 \quad$ World Journal 2012, 510149 DOI 10.1100/2012/510149.

483 Tura A, Sbrignadello S, Succurro E, Groop L, Sesti G, Pacini G. 2010. An empirical index of

484 insulin sensitivity from short IVGTT: validation against the minimal model and glucose 
485 clamp indices in patients with different clinical characteristics. Diabetologia 53, 144-152

486 DOI $10.1007 / \mathrm{s} 00125-009-1547-9$.

487 Wang X, Hu Z, Hu J, Du J, Mitch W. 2006. Insulin resistance accelerates muscle protein 488 degradation: activation of the ubiquitin-proteasome pathway by defects in muscle cell 489 signaling. Endocrinology 147, 4160-4168 DOI 10.1210/en.2006-0251.

490 Wangsness PJ, Acker WA, Burdette JH, Krabill LF, Vasilatos R. 1981. Effect of fasting on 491 hormones and metabolites in plasma of fast-growing, lean and slow-growing obese pigs. 492 Journal of Animal Science 52, 69-74 DOI 10.2527/jas1981.52169x.

493 Weiler U, Claus R, Schnoebelen Combes S, Louveau I. 1998. Influence of age and genotype on 494 endocrine parameters and growth performance: a comparative study in wild boars, Meishan 495 and Large White boars. Livestock Production Science 54, 21-31 DOI 10.1016/S0301$496 \quad 6226(97) 00165-6$.

497 Ykijarvinen H, Bogardus C, Foley JE. 1990. Regulation of plasma lactate concentration in 498 resting human-subjects. Metabolism-Clinical and Experimental 39, 859-864 DOI $499 \quad$ 10.1016/0026-0495(90)90133-W.

500 Zuur AF, Ieno EN, Elphick CS. 2010. A protocol for data exploration to avoid common $501 \quad$ statistical problems. Methods in Ecology and Evolution 1, 3-14 DOI 10.1111/j.2041$502 \quad$ 210X.2009.00001.x. 


\section{Table 1 (on next page)}

Average plasma metabolites and insulin concentrations in Iberian $(n=4)$ and Landrace $(n=5)$ pigs during an intra-arterial glucose challenge (IAGTT; $500 \mathrm{mg} / \mathrm{kg} \mathrm{BW,} \mathrm{0-180}$ $\min )^{a}$

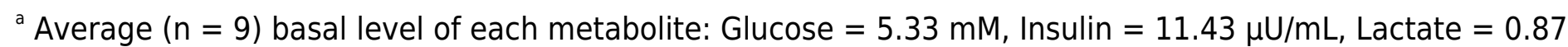
$\mathrm{mM}$, Triglycerides $=0.25 \mathrm{mM}$, Albumin $=0.46 \mathrm{mM}$, Cholesterol $=1.63 \mathrm{mM}$, Creatinine $=76.2 \mu \mathrm{M}$, Urea $=$ $2.92 \mathrm{mM}$.

${ }^{\mathrm{b}} \mathrm{ns}=$ non-significant, ${ }^{* *} P<0.01, * * * P<0.001$.

${ }^{\mathrm{c}}$ Standard error of mean. 


\begin{tabular}{|c|c|c|c|c|c|c|}
\hline & \multicolumn{2}{|l|}{ Breed } & \multirow[b]{2}{*}{ SEM $^{\mathrm{c}}$} & \multicolumn{3}{|c|}{$P$-value ${ }^{\mathrm{b}}$} \\
\hline & Iberian & Landrace & & Breed & Time & Breed $\times$ Time \\
\hline Insulin $(\mu \mathrm{U} / \mathrm{mL})$ & 41 & 27 & 1.9 & $* * *$ & $* * *$ & $* * *$ \\
\hline Glucose $(\mathrm{mmol} / \mathrm{L})$ & 6.8 & 7.7 & 0.26 & $* *$ & $* * *$ & ns \\
\hline Lactate $(\mathrm{mmol} / \mathrm{L})$ & 1.3 & 1.0 & 0.039 & $* * *$ & $* * *$ & ns \\
\hline Triglycerides $(\mathrm{mmol} / \mathrm{L})$ & 0.28 & 0.24 & 0.009 & $* *$ & ns & ns \\
\hline Cholesterol (mmol/L) & 1.5 & 1.8 & 0.033 & $* * *$ & ns & ns \\
\hline Creatinine $(\mu \mathrm{mol} / \mathrm{L})$ & 54 & 90 & 1.2 & $* *$ & ns & ns \\
\hline Albumin (mmol/L) & 0.48 & 0.50 & 0.009 & ns & ns & ns \\
\hline Urea $(\mathrm{mmol} / \mathrm{L})$ & 3.0 & 2.4 & 0.102 & $* * *$ & ns & ns \\
\hline
\end{tabular}




\section{Table 2 (on next page)}

Indices of glucose tolerance and insulin sensitivity in Iberian $(n=4)$ and Landrace $(n=$ 5) pigs subjected to an intra-arterial glucose tolerance test. ${ }^{a}$

${ }^{a}$ QUICKI, quantitative insulin sensitivity check index; HOMA-IR, homeostasis model assessment for estimating insulin resistance; $\mathrm{HOMA}-\% \mathrm{~B}$, homeostasis model assessment for estimating $\beta$-cell function; $\mathrm{CSI}$, calculated insulin sensitivity index.

${ }^{\mathrm{b}}$ Standard error of mean.

${ }^{\mathrm{c}} \mathrm{ns}=$ non-significant, ${ }^{\dagger} 0.05<P<0.10, * P<0.05, * * P<0.01$. 


\begin{tabular}{llllll}
\hline & Breed & & & \\
\cline { 2 - 3 } & Iberian & Landrace & SEM $^{\mathrm{b}}$ & $P$-value \\
\hline Fasting insulin $(\mu \mathrm{U} / \mathrm{mL})$ & 16 & 8 & 1.6 & $*$ \\
Fasting glucose $(\mathrm{mmol} / \mathrm{L})$ & 4.7 & 5.9 & 0.92 & $\mathrm{~ns}$ \\
Insulin disappearance rate $(\% / \mathrm{min})$ & 7.2 & 3.8 & 1.01 & $\dagger$ \\
Glucose disappearance rate $(\% / \mathrm{min})$ & 5.9 & 3.9 & 0.77 & $\dagger$ \\
Insulin half-live $(\mathrm{min})$ & 10 & 21 & 3.6 & $\dagger$ \\
Glucose half-live $(\mathrm{min})$ & 22 & 12 & 4.8 & $\mathrm{~ns}$ \\
QUICKI & 0.31 & 0.33 & 0.007 & $*$ \\
HOMA-IR & 3.3 & 2.3 & 0.58 & $\mathrm{~ns}$ \\
HOMA-\%B & 267 & 100 & 26 & $* *$ \\
CSI $\left(\times 10^{-4}\right)$ & -12 & -13 & 1.8 & $\mathrm{~ns}$ \\
\hline
\end{tabular}




\section{Table 3(on next page)}

Area under the curve (AUC, $\mu \mathrm{U} \times \mathrm{min} / \mathrm{mL}$ ) of plasma insulin during intra-arterial glucose tolerance test between minute 0 and indicated time post-challenge in Iberian $(n=4)$ and Landrace $(n=5)$ pigs.

${ }^{a}$ Standard error of mean.

${ }^{\mathrm{b}} * P<0.05, * * P<0.01$ 
1

\begin{tabular}{lllll}
\hline & Iberian & Landrace & SEM $^{\mathrm{a}}$ & $P$-value \\
\cline { 2 - 5 } AUC 0-5 min & 464 & 295 & 40.7 & $*$ \\
AUC 0-10 min & 993 & 587 & 72.6 & $* *$ \\
AUC 0-15 min & 1470 & 873 & 93.3 & $* *$ \\
AUC 0-20 min & 1768 & 1135 & 95.1 & $* *$ \\
AUC 0-25 min & 1919 & 1335 & 92.5 & $* *$ \\
AUC 0-30 min & 1993 & 1461 & 89.0 & $* *$ \\
AUC 0-45 min & 2169 & 1652 & 106.3 & $* *$ \\
AUC 0-60 min & 2340 & 1757 & 135.1 & $*$ \\
AUC 0-90 min & 2809 & 1985 & 176.3 & $*$ \\
AUC 0-120 min & 3358 & 2239 & 206.5 & $* *$ \\
AUC 0-150 min & 3787 & 2459 & 214.3 & $* *$ \\
AUC 0-180 min & 4183 & 2642 & 229.1 & $* *$ \\
\hline
\end{tabular}

2 


\section{Table 4 (on next page)}

Area under the curve (AUC, $\mathrm{mmol} \times \mathrm{min} / \mathrm{L}$ ) of plasma glucose during intra-arterial glucose tolerance test between minute 0 and indicated time post-challenge in Iberian ( $\mathrm{n}$ $=4)$ and Landrace $(n=5)$ pigs.

${ }^{a}$ Standard error of mean.

${ }^{\mathrm{b}} \mathrm{ns}=$ non-significant, ${ }^{\dagger} 0.05<P<0.10, * P<0.05$ 
1

\begin{tabular}{lllll}
\hline & Iberian & Landrace & SEM $^{\mathrm{a}}$ & $P$-value \\
\cline { 2 - 5 } AUC 0-5 min & 82.6 & 90.8 & 3.94 & $\mathrm{~ns}$ \\
AUC 0-10 min & 140 & 159 & 7.6 & $\dagger$ \\
AUC 0-15 min & 181 & 215 & 10.4 & $*$ \\
AUC 0-20 min & 211 & 260 & 12.9 & $*$ \\
AUC 0-25 min & 235 & 295 & 15.7 & $*$ \\
AUC 0-30 min & 252 & 323 & 18.7 & $*$ \\
AUC 0-45 min & 297 & 388 & 25.7 & $*$ \\
AUC 0-60 min & 350 & 446 & 28.3 & $*$ \\
AUC 0-90 min & 489 & 575 & 30.4 & $\dagger$ \\
AUC 0-120 min & 631 & 710 & 30.0 & $\dagger$ \\
AUC 0-150 min & 769 & 845 & 30.2 & $\mathrm{~ns}$ \\
AUC 0-180 min & 913 & 979 & 31.7 & $\mathrm{~ns}$ \\
\hline
\end{tabular}

2 


\section{Table 5 (on next page)}

Area under the curve (AUC, $\mathrm{mmol} \times \mathrm{min} / \mathrm{L}$ ) of plasma lactate during intra-arterial glucose tolerance test between minute 0 and indicated time post-challenge in Iberian ( $\mathrm{n}$ $=4)$ and Landrace $(n=5)$ pigs.

${ }^{a}$ Standard error of mean.

${ }^{\mathrm{b}} \mathrm{ns}=$ non-significant, ${ }^{\dagger} 0.05<P<0.10, * P<0.05$. 
1

\begin{tabular}{lllll}
\hline & Iberian & Landrace & SEM $^{\mathrm{a}}$ & $P$-value \\
\cline { 2 - 5 } AUC 0-5 min & 5.81 & 4.34 & 0.473 & $\dagger$ \\
AUC 0-10 min & 12.8 & 9.4 & 0.91 & $*$ \\
AUC 0-15 min & 20.7 & 15.5 & 1.45 & $*$ \\
AUC 0-20 min & 29.0 & 22.1 & 2.23 & $\dagger$ \\
AUC 0-25 min & 37.3 & 28.9 & 3.09 & $\dagger$ \\
AUC 0-30 min & 45.4 & 35.6 & 4.00 & $\mathrm{~ns}$ \\
AUC 0-45 min & 68.4 & 53.3 & 6.87 & $\mathrm{~ns}$ \\
AUC 0-60 min & 89.6 & 67.1 & 9.29 & $\mathrm{~ns}$ \\
AUC 0-90 min & 123 & 89.4 & 13.01 & $\dagger$ \\
AUC 0-120 min & 152 & 109 & 15.7 & $\dagger$ \\
AUC 0-150 min & 180 & 128 & 17.6 & $\dagger$ \\
AUC 0-180 min & 208 & 145 & 20.7 & $\dagger$ \\
\hline
\end{tabular}

2 
Figure 1

Plasma insulin concentration during intra-arterial glucose challenge test $(500 \mathrm{mg} / \mathrm{kg}$ BW; 180 min sampling) in growing Iberian $(n=4)$ and Landrace $(n=5)$ pigs.

${ }^{\dagger} 0.05<P<0.10, * P<0.05, * * * P<0.001$.

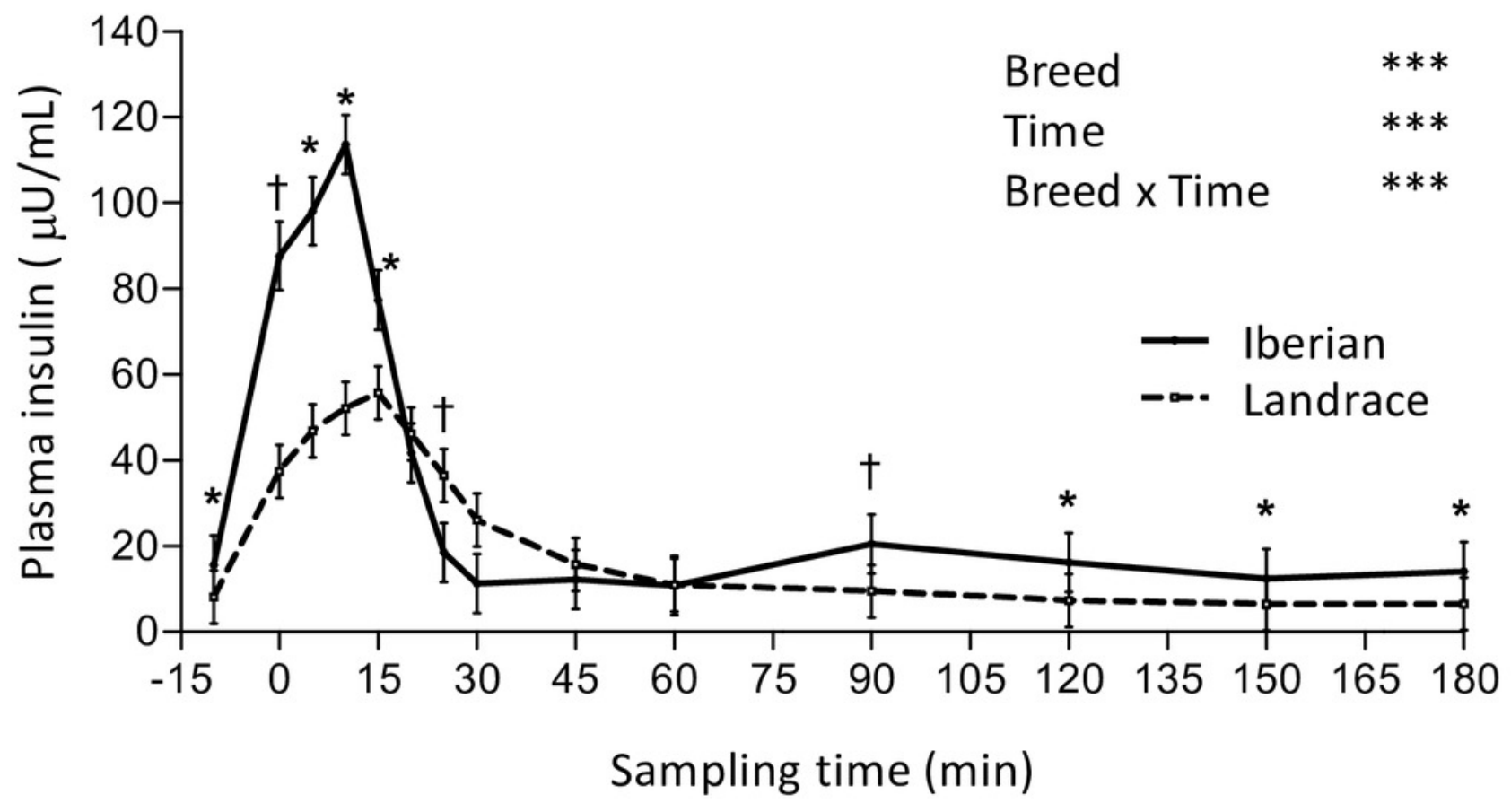


Figure 2

Plasma glucose concentration during intra-arterial glucose challenge test $(500 \mathrm{mg} / \mathrm{kg}$ BW; 180 min sampling) in growing Iberian $(n=4)$ and Landrace $(n=5)$ pigs.

ns, not significant $(P>0.10) ;{ }^{\dagger} 0.05<P<0.10, * P<0.05, * * P<0.01, * * * P<0.001$.

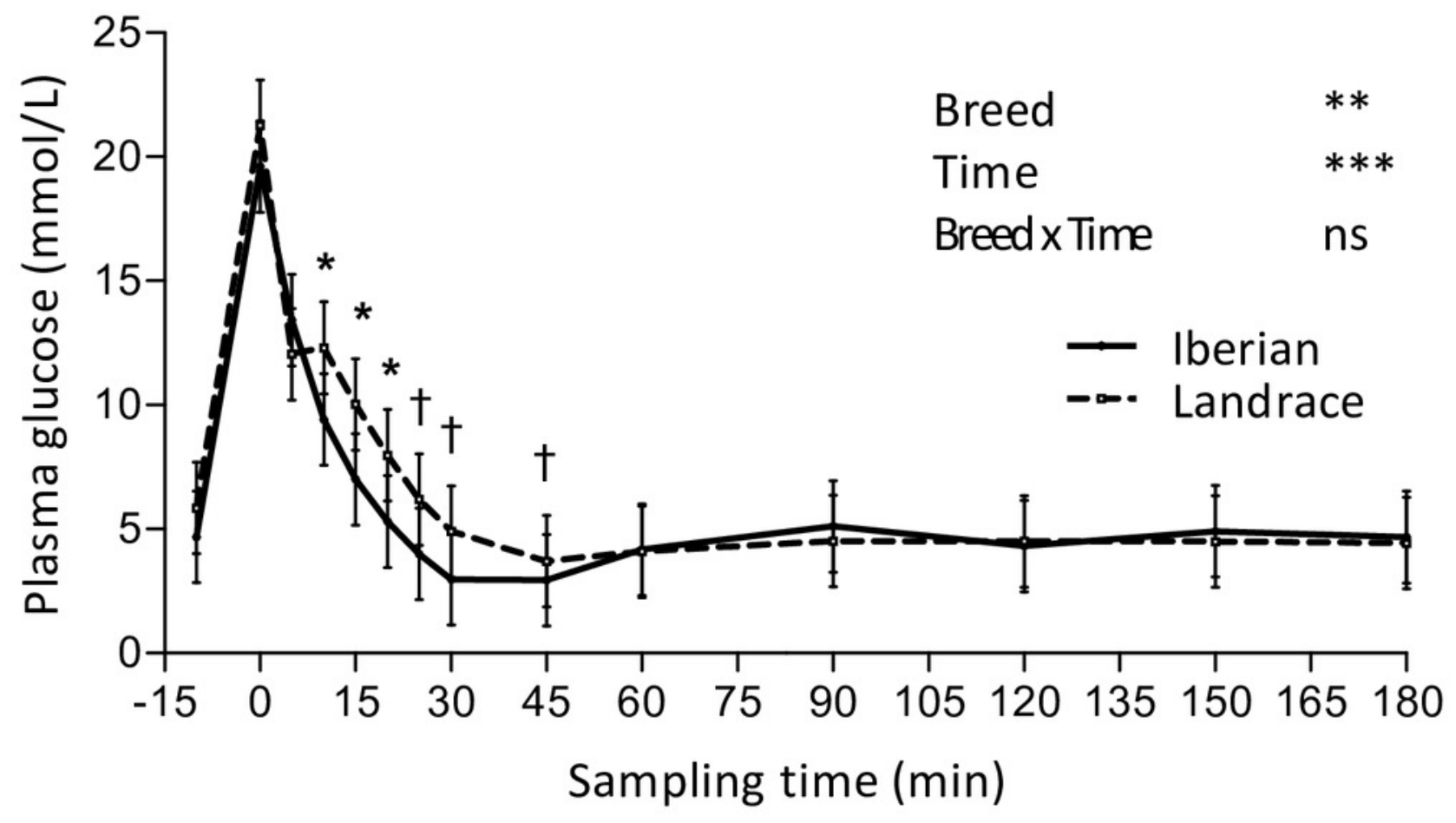


Figure 3

Plasma lactate concentration during intra-arterial glucose tolerance test $(500 \mathrm{mg} / \mathrm{kg}$ BW; 180 min sampling) in growing Iberian $(n=4)$ and Landrace $(n=5)$ pigs.

ns, not significant $(P>0.10) ;{ }^{\dagger} 0.05<P<0.10, * P<0.05$, *** $P<0.001$.

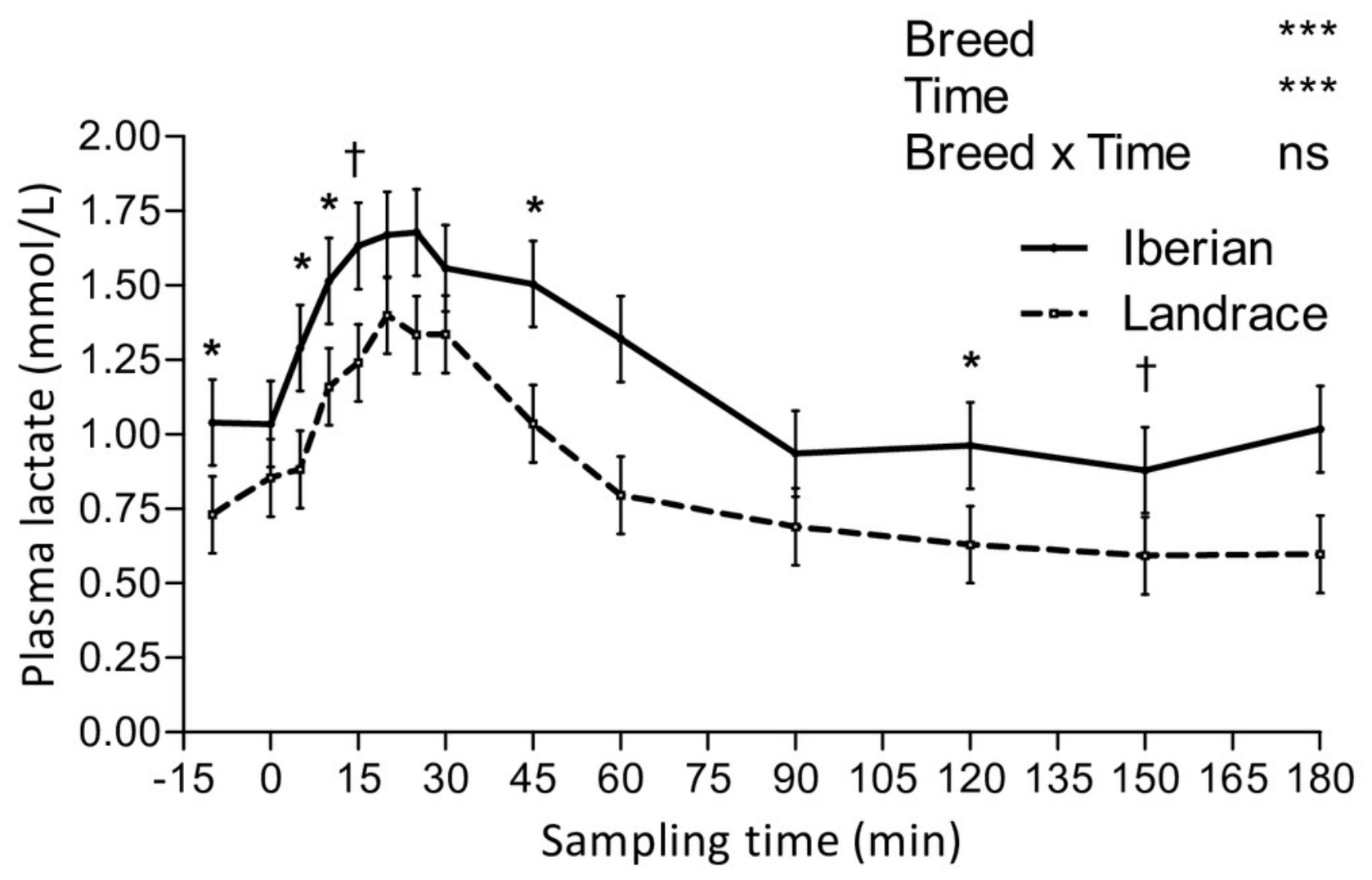

